\title{
Biological Dose Comparison between a Fixed RBE and a Variable RBE in SFO and MFO IMPT with Various Multi-Beams for Brain Cancer
}

\author{
Ryosuke Kohno1,2,3, Wenhua Cao², Pablo Yepes ${ }^{2}$, Xuemin Bai², Falk Poenisch', \\ David R. Grosshans ${ }^{2}$, Tetsuo Akimoto ${ }^{3}$, Radhe Mohan² \\ ${ }^{1}$ Medical Physics Section, National Institute of Radiological Sciences, Chiba, Japan \\ ${ }^{2}$ Department of Radiation Physics, University of Texas MD Anderson Cancer Center, Houston, USA \\ ${ }^{3}$ Division of Radiation Oncology and Particle Therapy, Exploratory Oncology Research \& Clinical Trial Center, \\ National Cancer Center Hospital East, Chiba, Japan \\ Email: kohno.ryosuke@qst.go.jp
}

How to cite this paper: Kohno, R., Cao, W.H., Yepes, P., Bai, X.M., Poenisch, F., Grosshans, D.R., Akimoto, T. and Mohan, R. (2019) Biological Dose Comparison between a Fixed RBE and a Variable RBE in SFO and MFO IMPT with Various Multi-Beams for Brain Cancer. International Journal of Medical Physics, Clinical Engineering and Radiation Oncology, 8, 32-45. https://doi.org/10.4236/ijmpcero.2019.8100 $\underline{4}$

Received: November 28, 2018

Accepted: January 30, 2019

Published: February 2, 2019

Copyright $\odot 2019$ by author(s) and Scientific Research Publishing Inc. This work is licensed under the Creative Commons Attribution International License (CC BY 4.0). http://creativecommons.org/licenses/by/4.0/

\begin{abstract}
IMPT plans with various multi-angle beams were planned by the Varian Eclipse treatment planning system for one case of brain cancer. Dose distributions for each plan, along with the associated linear energy transfer distributions, were recomputed using an in-house fast Monte Carlo dose calculator with a FRBE of 1.1 or with a previously published VRBE model. We then compared dosimetric parameters obtained by the VRBE with those obtained by the FRBE. Biological doses obtained by the VRBE for the clinical target volume in all plans were $1 \%-2 \%$ larger than those obtained by the FRBE. The minimum dose obtained by the VRBE for the right optic nerve in the MFO IMPT with 4 fields was $70 \%$ larger than that obtained by the FRBE, but the difference was only $18.1 \mathrm{cGy}$ (RBE). The difference in maximum dose for the right optic nerve in the MFO IMPT with 5 fields was less than $10.4 \%$, but the difference was $131.8 \mathrm{cGy}$ (RBE). The mean difference in maximum dose was less than $2 \%$ for all other organs at risk. We found that biological dose with the FRBE had any dose errors in IMPT with various multi-angle beams.
\end{abstract}

\section{Keywords}

IMPT, Monte Carlo, Biological Dose, Variable RBE

\section{Introduction}

Proton beam therapy can enhance tumor control while minimizing irradiation to surrounding normal tissues in cancer care owing to the Bragg peak, with a sharp distal fall-off [1] [2]. Recently, the number of clinical proton therapy facil- 
ities has increased because of this substantial potential for clinical advantages over conventional photon therapy [3] [4]. In the latest proton beam therapy, beam scanning technique [5] [6] [7] has been widely used. Especially, intensity-modulated proton therapy (IMPT), in which scanning beam lets of protons, used to "paint" radiation dose on the target, can be exploited to safely bend beams around complex critical structures, allowing improved sparing of these structures without compromising target coverage [8]. Two types of IMPT optimization exist, namely, single-field optimization (SFO) and multi-field optimization (MFO). The SFO optimizes individually each field to create a uniform dose distribution from each beam, and the MFO optimizes simultaneously spots from all the fields to get highly conformal dose distributions.

Currently, a fixed relative biological effectiveness (FRBE) relative to photons of 1.1 is used conventionally in proton beam therapy. However, the RBE of protons generally depends on the linear energy transfer (LET), the dose per fraction, and the tissue type [9]. In recent years, the potential clinical impacts of a variable RBE (VRBE) in proton beam therapy have been discussed [10] [11] [12] [13]. Frese et al. have investigated whether using a VRBE in IMPT will change dose distributions [14]. They indicate that the application of the VRBE is clinically more useful for accurate assessment of the feasibility of the plans from a biological point of view.

However, in that study, Wilkens and Oelfke use a pencil beam algorithm (PBA) to simulate dose and LET distributions [15]. It is common knowledge that the accuracy of the PBA [16] for protons is inadequate, especially in the presence of complex heterogeneities [17] [18]. In the SFO and MFO IMPT plans with various multi-angle beams for a brain cancer patient, physical dose differences between the PBA and Monte Carlo method have been already evaluated, and Kohno et al. also advocate use of a Monte Carlo method in proton treatment planning to deliver the most precise proton dose in IMPT [19].

In this study, dose distributions, along with the associated linear energy transfer (LET) distributions, were recomputed using a fast Monte Carlo dose calculator (FDC). We focused on biological dose differences between FRBE and VRBE for the same SFO and MFO IMPT plans with various multi-angle beams in the previous paper [19]. From these studies, we expect that it is useful for proton users to clarify dose differences of not only physical dose calculation algorithms but also biological dose calculation models.

\section{Materials and Methods}

We selected a patient with grade 3 anaplastic astrocytoma analyzed by Kohno et al. [19]. This patient was treated by passively scattered proton therapy at The University of Texas MD Anderson Cancer Center. Figure 1 shows the target volumes and organs at risk (OARs) in the brain cancer treatment plan on a computed tomography slice. The target volumes are surrounded in a complicated manner by nerve, brain, brainstem, bone, and sinus cavity. As shown in 


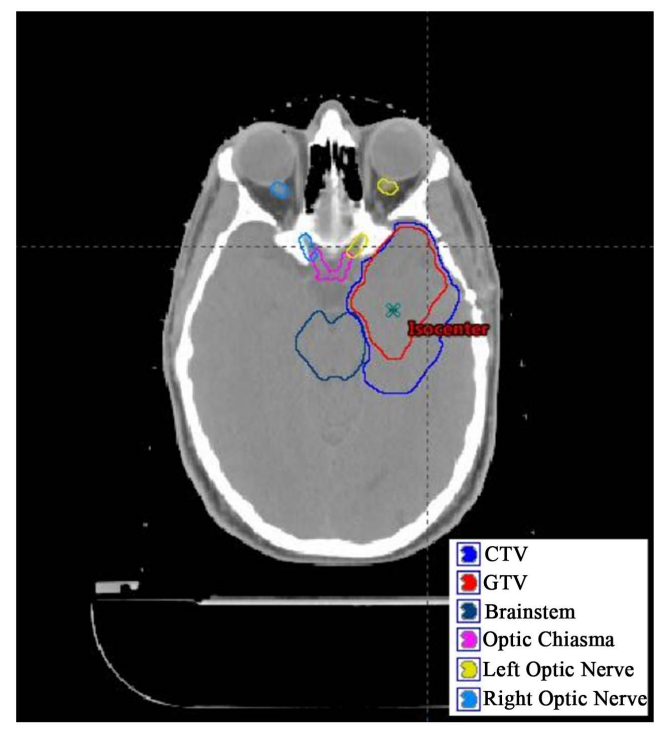

Figure 1. Contours of the GTV, CTV, and OARs drawn on the computed tomography image for the actual brain cancer IMPT plan analyzed by Kohno et al. [19].

Table 1, we used the SFO and MFO IMPT plans with three, four, five, six, or nine treatment fields analyzed by Kohno et al. [19].

SFO and MFO IMPT plans were designed by the Eclipse treatment planning system (Version 13.5; Varian Medical Systems, Inc., Palo Alto, CA). The prescribed doses were $5700 \mathrm{cGy}$ (RBE) to the gross tumor volume (GTV) and 5000 cGy (RBE) to the clinical target volume (CTV) in 30 fractions. All plans were designed to cover $100 \%$ of the GTV and the CTV and to minimize the maximum dose for each OAR with the same optimization conditions as in the treatment planning system. The maximum dose constraint for the brainstem, the chiasm, left optic nerve, and right optic nerve was $5400 \mathrm{cGy}$ (RBE).

The FDC [20] [21] recomputes physical dose distributions for each plan, along with the associated LET distributions. Then, to calculate a biological dose distribution, RBE was obtained by the FRBE of 1.1 and the phenomenological RBE model [22]. This phenomenological model can calculate simply the RBE as a function of the dose, the LET, and tissue-specific parameters. Using a reference radiation with parameters $\alpha_{x}$ and $\beta_{x}$, the RBE at proton dose $\left(D_{p}\right)$ on the dose averaged LET ( $\left.L E T_{d}\right)$ is given by

$$
R B E\left(D_{p}, L E T_{d}, \alpha_{0}, \lambda, \alpha_{x}, \beta_{x}\right)=\frac{\sqrt{\alpha_{x}^{2}+4 \beta_{x} D_{p}\left(\alpha_{0}+\lambda L E T_{d}+\beta_{x} D_{p}\right)}-\alpha_{x}}{2 \beta_{x} D_{p}}
$$

where $\alpha_{0}$ and $\lambda$ are the linear and the initial parameter, respectively, in terms of biologic response $(\alpha)$ to protons. Biological parameters given by Frese et al. [14] were used in this study.

To compare the FRBE and VRBE models, we performed a comparative analysis of dose distributions for each plan in each region of interest. The comparative analysis used the dosimetric parameters of minimum, maximum, and mean dose. For the CTV, we also evaluated dose to $95 \%$ of the CTV (D95), dose to 
Table 1. Treatment fields and beam angles (degrees) for each plan.

\begin{tabular}{|c|c|c|c|c|c|c|c|c|c|c|c|}
\hline \multicolumn{2}{|c|}{ Plan } & \multirow{2}{*}{$\begin{array}{c}\text { Number of } \\
\text { treatment } \\
\text { fields }\end{array}$} & \multirow{2}{*}{\multicolumn{9}{|c|}{ Beam angle (degrees) }} \\
\hline SFO & MFO & & & & & & & & & & \\
\hline A & G & 3 & 40 & 100 & 160 & NA & NA & NA & NA & NA & NA \\
\hline B & $\mathrm{H}$ & 4 & 40 & 80 & 120 & 160 & NA & NA & NA & NA & NA \\
\hline $\mathrm{C}$ & I & 5 & 40 & 70 & 100 & 130 & 160 & NA & NA & NA & NA \\
\hline $\mathrm{D}$ & $\mathrm{J}$ & 6 & 40 & 70 & 100 & 130 & 160 & 220 & NA & NA & NA \\
\hline $\mathrm{E}$ & $\mathbf{K}$ & 9 & 40 & 70 & 100 & 130 & 160 & 190 & 220 & 250 & 280 \\
\hline F & L & 9 & 20 & 60 & 100 & 140 & 180 & 220 & 260 & 300 & 340 \\
\hline
\end{tabular}

$5 \%$ of the CTV (D5), heterogeneity index (HI), and conformity index (CI). As defined by the Radiation Therapy Oncology Group [23], HI is the maximum dose to the CTV divided by the prescription dose, and CI is the prescription isodose volume divided by the CTV volume.

\section{Results}

The SFO IMPT plans obtained by the FRBE and by the VRBE models differed by a mean ( \pm standard deviation) of $1.5 \% \pm 0.3 \%$ in maximum dose and $1.3 \% \pm$ $0.0 \%$ in mean dose (Figure 2). In order to observe easily dependence of dose on number of fields, all data was fitted with linear function. The difference between the models in minimum dose was somewhat larger, at $2.2 \% \pm 0.4 \%$. These results did not depend on number of fields or on plans. The FRBE and the VRBE models differed by a mean ( \pm standard deviation) of $1.4 \% \pm 0.1 \%$ in D95 and $1.3 \% \pm 0.1 \%$ in D5. Overall, doses obtained by the VRBE were about $2 \%$ larger than those obtained by the FRBE. The FRBE and VRBE models differed by $1.6 \%$ $\pm 0.3 \%$ in $\mathrm{HI}$ and $3.9 \% \pm 0.4 \%$ in $\mathrm{CI}$, and both indexes in the VRBE deteriorated compared with those in the FRBE.

Minimum, maximum, and mean dose obtained by the FRBE and the VRBE models in the SFO IMPT plans for the OARs are shown in Figure 3. For each site, the differences in minimum dose were larger than the differences in maximum and mean dose. The difference in minimum dose in the right optic nerve in plan B was the largest among the plans, at $56.4 \%$, but the dose difference was only $21.7 \mathrm{cGy}$ (RBE). The maximum dose to these critical organs is the most important parameter; the difference in maximum dose in the right optic nerve in plan $C$ was less than $7.7 \%$ but constituted a difference of 111.6 cGy (RBE). This maximum dose was much less than the maximum dose constraint of $5400 \mathrm{cGy}$ (RBE). Except for the right optic nerve, the mean difference in maximum dose for each site was less than $2 \%$.

Figure 4 shows the minimum, maximum, and mean LET in the SFO IMPT plans obtained by the FRBE and VRBE models at different locations. The LET in the brainstem ranged from 1.2 to $7.7 \mathrm{keV} / \mu \mathrm{m}$ (the widest range), while the LET in the left optic nerve ranged from 2.3 to $5.5 \mathrm{keV} / \mu \mathrm{m}$ (the narrowest range). Overall, LET decreased with number of fields because the ends of the beams with high LETs did not concentrate at each site, owing to multiple beam angles. 

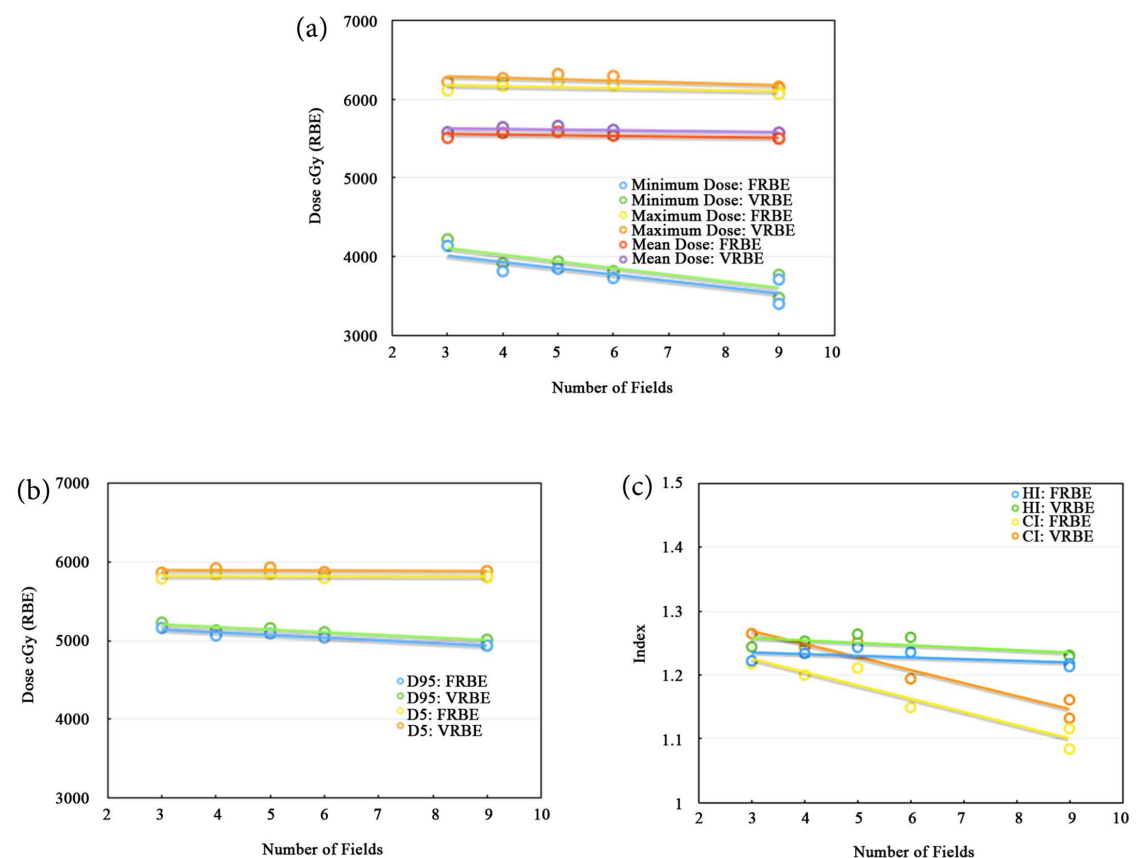

Figure 2. (a) Minimum, maximum, and mean dose; (b) D95 and D5; (c) HI and CI for the CTV obtained by the FRBE and the VRBE models in SFO IMPT plans.
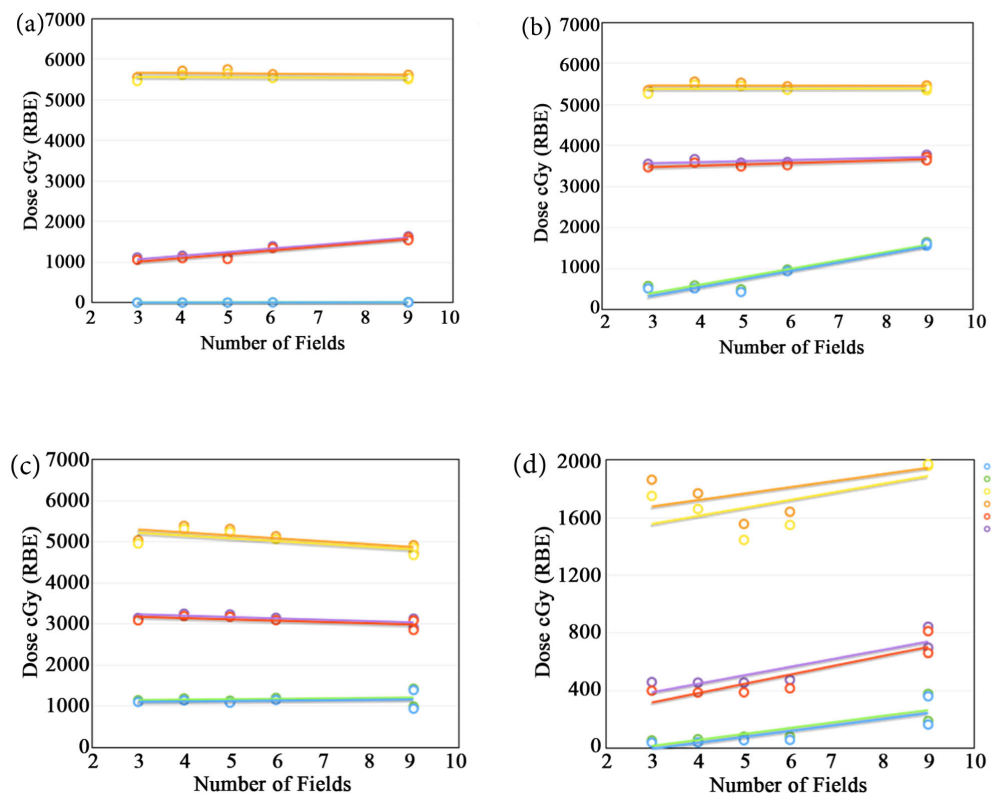

Figure 3. Minimum, maximum, and mean dose in SFO IMPT plans obtained by the FRBE and VRBE models for the (a) brainstem; (b) chiasm; (c) left optic nerve; (d) right optic nerve.

The dosimetric parameters for the CTV in the MFO IMPT plans differed on average between the FRBE and the VRBE models in minimum, maximum, and mean dose by $1.2 \% \pm 0.5 \%, 1.6 \% \pm 0.5 \%$, and $1.1 \% \pm 0.2 \%$, respectively (Figure 5). These differences did not depend on plan. The mean differences between the models in D95 and D5 were $1.2 \% \pm 0.2 \%$ and $1.0 \% \pm 0.2 \%$, respectively. The 

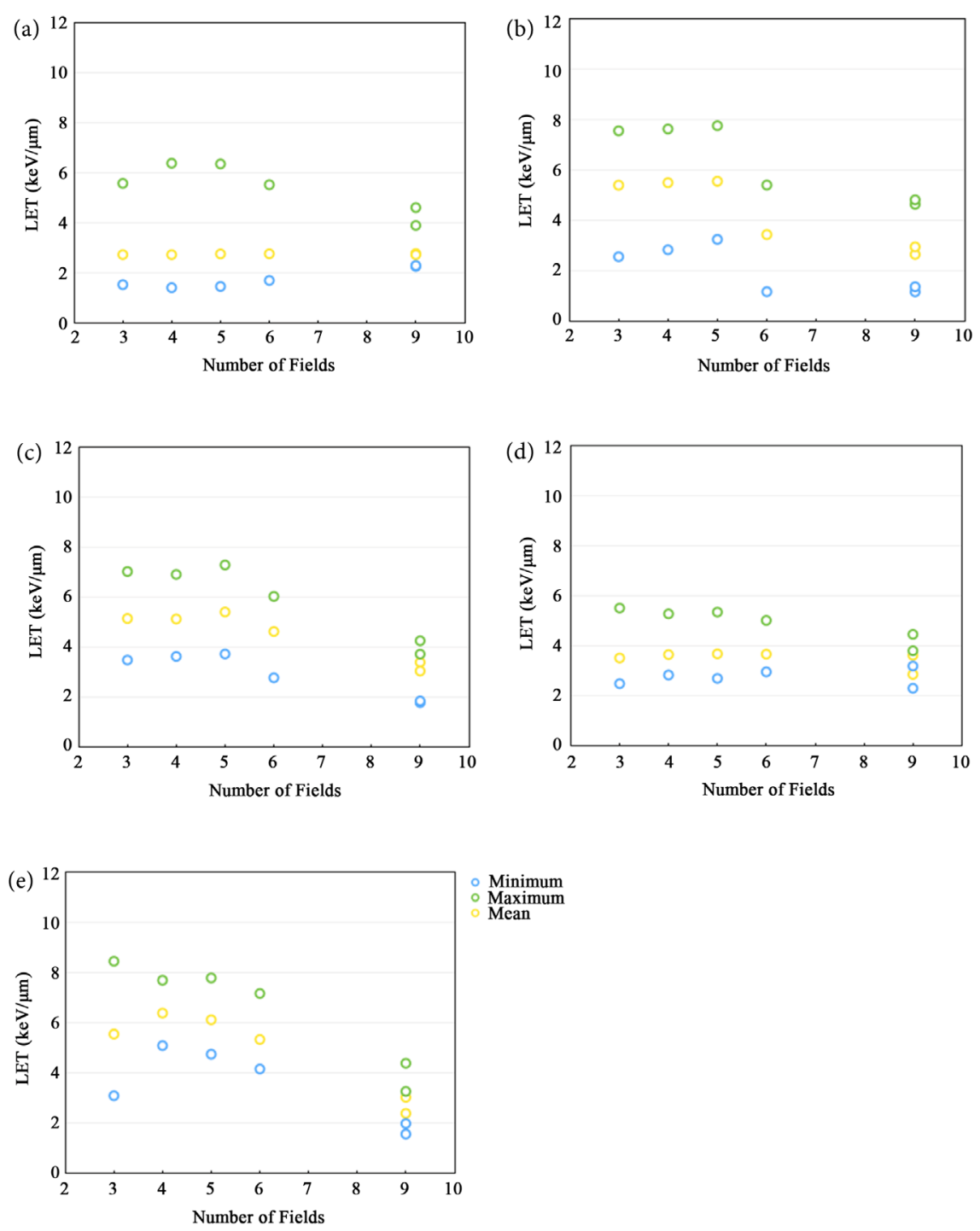

Figure 4. Minimum, maximum, and mean LET in SFO IMPT plans obtained by the FRBE and VRBE models for the (a) CTV; (b) brainstem; (c) chiasm; (d) left optic nerve; (e) right optic nerve.

mean differences between the models in HI and CI were $1.6 \% \pm 0.5 \%$ and $4.2 \% \pm$ $0.5 \%$, respectively. Both indexes in the VRBE model deteriorated compared with those in the FRBE, similar to the indexes in the SFO IMPT plans. There was almost no difference between the results for the SFO plans and the results for the MFO plans.

Minimum, maximum, and mean dose in the MFO IMPT plans obtained by the FRBE and VRBE models for the OARs are shown in Figure 6. The differences in minimum dose were larger than the differences in maximum dose and the differences in mean dose for each site, and the differences in each parameter in the right optic nerve were larger than those in other sites. As Figure 6(d) shows, differences in each dosimetric parameter decreased with number of fields. Their differences in plan $\mathrm{L}$ were minimal in all plans. The difference in 

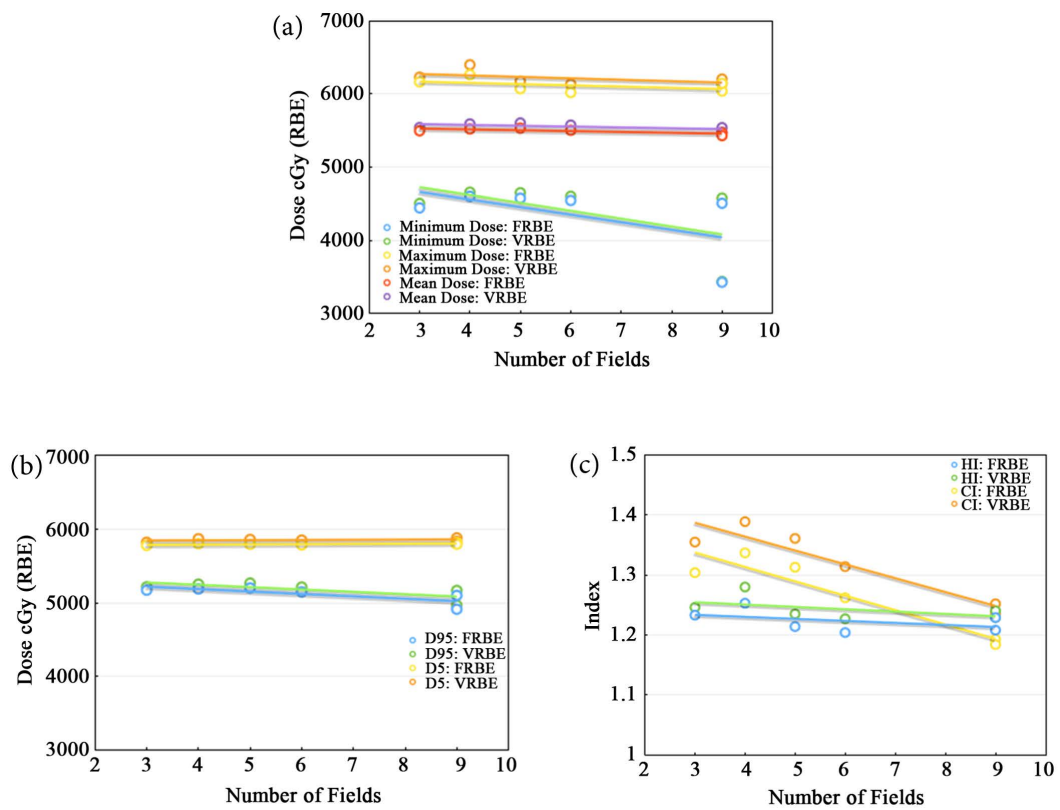

Figure 5. (a) Minimum, maximum, and mean dose; (b) D95 and D5; and (c) HI and CI for the CTV obtained by the FRBE and VRBE models in MFO IMPT plans.
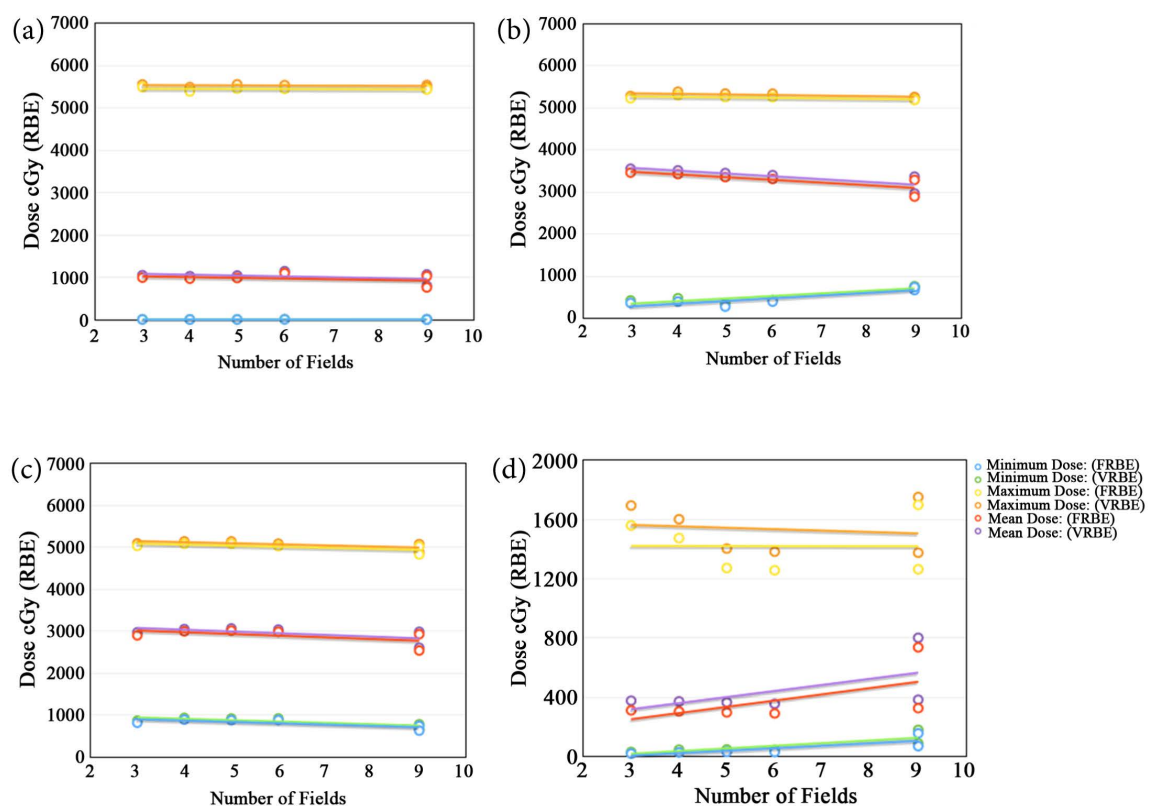

Figure 6. Minimum, maximum, and mean dose obtained by the FRBE and VRBE models in MFO IMPT plans for the (a) brainstem; (b) chiasm; (c) left optic nerve; (d) right optic nerve.

minimum dose to the right optic nerve in plan $\mathrm{H}$ was the largest, at $70.4 \%$, but this difference was only $18.1 \mathrm{cGy}(\mathrm{RBE})$. The difference in the important parameter of maximum dose in the right optic nerve in plan I was less than $10.4 \%$, but this difference was only $131.8 \mathrm{cGy}$ (RBE). Except for the right optic nerve, the mean difference in maximum dose was less than $2 \%$ for each site, similar to the results of the SFO IMPT plans. 
Figure 7 shows the minimum, maximum, and mean LET in the MFO IMPT plans obtained by the FRBE and VRBE models at different locations. The LET in the right optic nerve ranged from 0.2 to $10.5 \mathrm{keV} / \mu \mathrm{m}$, and the minimum LET of 0.2 was obtained by secondary particles. The LET values in the brainstem and right optic nerve deposited mainly by the end of the beam showed wide ranges. Also, the range of the LET in the MFO plans was larger than that in the SFO plans (Figure 4).

Table 2 summarizes, for better comparison, the mean differences (cGy [RBE], \%) between the FRBE and the VRBE models in minimum, maximum and mean dose in the SFO and MFO IMPT plans for the CTV and OARs. For the $\mathrm{CTV}$, the mean differences in minimum, maximum and mean dose were $1 \%$ $2 \%$. For each OAR, the mean difference in maximum dose was the smallest
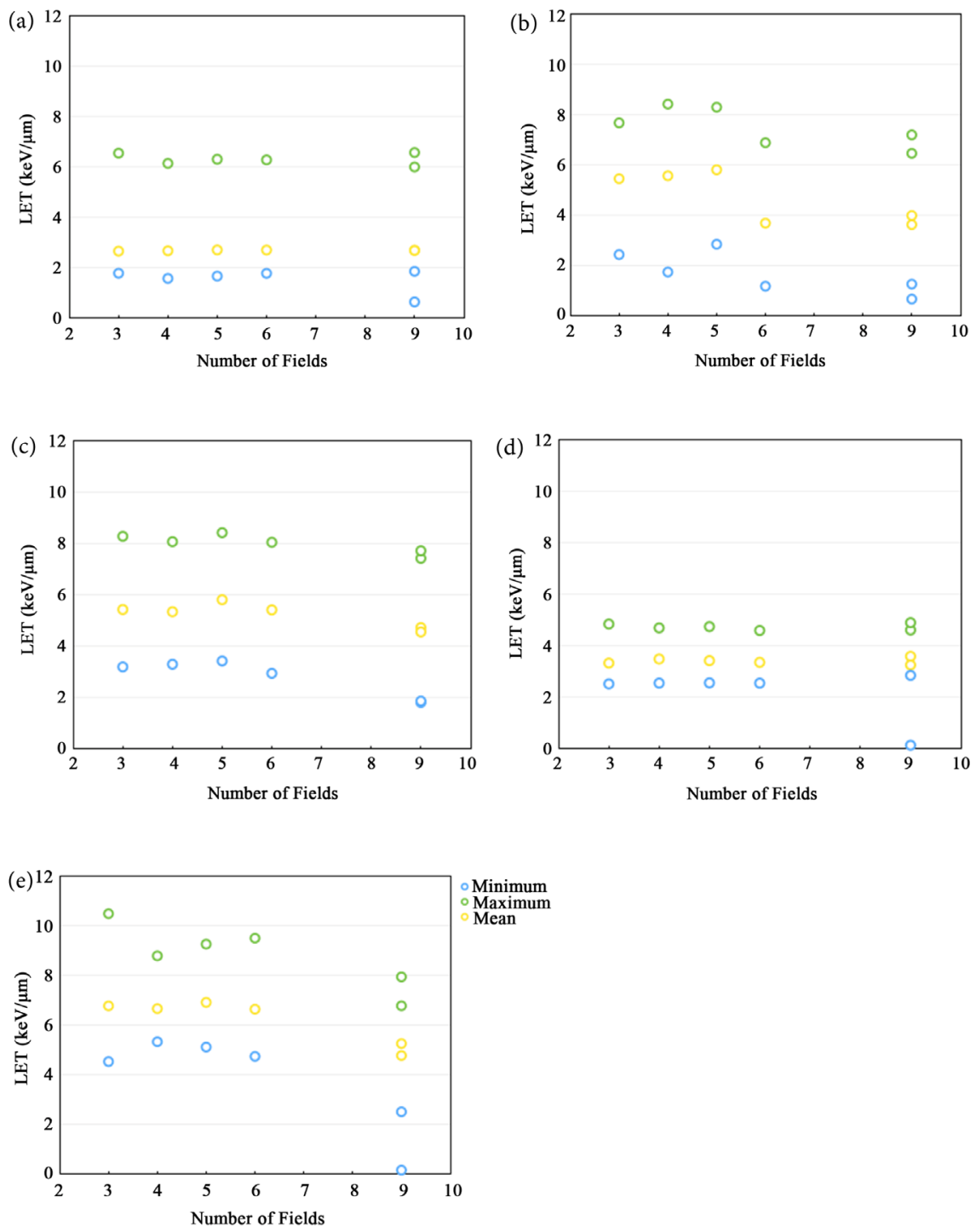

Minimum Maximum
Mean

Figure 7. Minimum, maximum, and mean LET in MFO IMPT plans obtained by the FRBE and VRBE models for the (a) CTV; (b) brainstem; (c) chiasm; (d) left optic nerve; (e) right optic nerve. 
Table 2. Mean differences between the FRBE and the VRBE models in dosimetric parameters for the SFO and MFO IMPT plans for each site.

\begin{tabular}{|c|c|c|c|c|c|c|c|}
\hline \multirow{2}{*}{$\begin{array}{c}\text { IMPT } \\
\text { type }\end{array}$} & \multirow{2}{*}{ Site } & \multicolumn{2}{|c|}{ Minimum dose } & \multicolumn{2}{|c|}{ Maximum dose } & \multicolumn{2}{|c|}{ Mean dose } \\
\hline & & cGy (RBE) & $\%$ & cGy (RBE) & $\%$ & cGy (RBE) & $\%$ \\
\hline \multirow{5}{*}{ SFO } & CTV & $84.1 \pm 14.4$ & $2.2 \pm 0.4$ & $95.1 \pm 18.3$ & $1.5 \pm 0.3$ & $71.6 \pm 0.7$ & $1.3 \pm 0.0$ \\
\hline & Brainstem & $3.7 \pm 0.8$ & $42.3 \pm 8.6$ & $91.6 \pm 1.6$ & $1.6 \pm 0.2$ & $48.5 \pm 13.2$ & $4.0 \pm 1.7$ \\
\hline & Chiasma & $54.2 \pm 15.2$ & $8.5 \pm 6.0$ & $75.6 \pm 8.1$ & $1.4 \pm 0.2$ & $74.2 \pm 18.0$ & $2.1 \pm 0.5$ \\
\hline & Left optic nerve & $45.4 \pm 7.0$ & $4.1 \pm 1.0$ & $67.5 \pm 11.6$ & $1.3 \pm 0.2$ & $56.3 \pm 6.8$ & $1.8 \pm 0.2$ \\
\hline & Right optic nerve & $20.8 \pm 4.0$ & $33.9 \pm 20.0$ & $88.7 \pm 28.2$ & $5.4 \pm 2.1$ & $53.8 \pm 15.8$ & $12.3 \pm 6.0$ \\
\hline \multirow{5}{*}{ MFO } & CTV & $54.0 \pm 23.1$ & $1.2 \pm 0.5$ & $96.4 \pm 29.4$ & $1.6 \pm 0.5$ & $61.4 \pm 12.8$ & $1.1 \pm 0.2$ \\
\hline & Brainstem & $2.4 \pm 1.3$ & $38.9 \pm 14.7$ & $81.1 \pm 21.0$ & $1.5 \pm 0.4$ & $50.3 \pm 9.1$ & $5.2 \pm 0.9$ \\
\hline & Chiasma & $56.0 \pm 22.5$ & $13.9 \pm 7.7$ & $68.4 \pm 14.1$ & $1.3 \pm 0.3$ & $86.6 \pm 8.4$ & $2.6 \pm 0.2$ \\
\hline & Left optic nerve & $40.9 \pm 4.6$ & $5.1 \pm 0.9$ & $58.4 \pm 2.1$ & $1.2 \pm 0.1$ & $60.0 \pm 8.1$ & $2.1 \pm 0.4$ \\
\hline & Right optic nerve & $16.7 \pm 4.3$ & $46.1 \pm 21.0$ & $114.1 \pm 30.8$ & $8.3 \pm 2.6$ & $64.8 \pm 4.1$ & $19.3 \pm 5.6$ \\
\hline
\end{tabular}

among those in minimum, maximum and mean dose. In maximum dose, except for the right optic nerve, the mean differences for all other OARs were less than $2 \%$. On the other hand, the mean difference in minimum dose for each OAR was the largest among them. Namely, minimum dose in all plans for OARs was changed sensitively by the VRBE calculation. Then, the mean differences in each dosimetric parameter for the right optic nerve in the SFO plans were smaller than those in the MFO plans. This is because LETs in the SFO were lower than those in the MFO, as shown in LET comparisons between Figure 4 and Figure 7.

\section{Discussion}

We observed dose differences between the plans obtained by the FRBE and VRBE models. Doses obtained by the VRBE for the CTV in all plans were 1\% $2 \%$ larger than those obtained by the FRBE. The differences between the models in each dosimetric parameter for the CTV in all plans did not depend on number of fields or on plans.

On the other hand, the minimum dose obtained by the VRBE for the right optic nerve in MFO plan $\mathrm{H}$ was $70 \%$ larger than that obtained by the FRBE, although the mean differences in maximum dose were less than $2 \%$ for all other OARs. The differences in each dosimetric parameter between the FRBE and VRBE models in the right optic nerve also decreased with the number of fields (Figure 6(d)). This decrease can be explained by differences in the right optic nerve between the maximum LET and the minimum LET, which decreased with the number of fields (Figure 7).

Thus, biological dose differences depended on location of the OAR, and LET 
decreased with number of fields because the ends of the beams with high LETs did not concentrate at each site owing to multiple beam angles. These results may lead to improvements of proton arc therapy with the ultimate multiple-field irradiation [24] [25] [25]. Furthermore, this knowledge may enhance the effectiveness of proton therapy by optimizing true biological dose [9].

In this study, the phenomenological RBE model proposed by Wilkens and Oelfke was used. Dose distributions with the VRBE naturally depend on the RBE calculation model. Although many researchers have also proposed RBE models [27] [28] [29], additional studies will be needed to accurately determine the dependence of RBE on various physical and biologic parameters [30] [31].

Regardless, we were able to evaluate the importance of a VRBE in this study. Dose differences between the FRBE and the VRBE models for the CTV were only about $2 \%$. On the other hand, Kohno et al. reported that the conventional pencil beam algorithm (PBA) dose calculation overestimated 400 - 500 cGy (RBE) for minimum physical dose to the CTV relative to the physical dose calculated by the FDC [19]. Mizutani et al. also reported that the D95 for PTV obtained by the simplified Monte Carlo method [32] [33] was 25\% smaller than that obtained by conventional pencil beam algorithm (PBA) dose calculation [34]. Thus, for the target volume, these results indicate that the dose errors by the physical dose calculation model would be greater in current proton beam therapy than those calculated by the RBE calculation model. Therefore, we strongly suggest that proton treatment planning and IMPT optimization use a fast Monte Carlo method such as the FDC or the simplified Monte Carlo method in advance.

\section{Conclusions}

In our evaluation of the dosimetric impacts of FRBE and VRBE models in SFO and MFO IMPT plans with various multi-angle beams using the FDC for a brain cancer patient, doses obtained by the VRBE model for CTV in all plans were about $2 \%$ larger than those obtained by the FRBE model. These differences were not large, and the differences among the models in each dosimetric parameter for the CTV in all plans did not depend on plans. On the other hand, for right optic nerve in plan $\mathrm{H}$, minimum and maximum dose obtained by the VRBE model were about $70 \%$ and $10 \%$ larger than that by the FRBE, and the mean difference in maximum dose for all other OARs was less than $2 \%$.

In conclusion, we found that biological dose with the FRBE had any dose errors in IMPT with various multi-angle beams. Minimum dose in all plans for OARs was changed sensitively by the VRBE calculation. This study indicated that the VRBE should be considered for proton treatment planning to provide an optimal proton beam therapy. However, in order to determine definitely whether there is any clinical evidence in which IMPT plans can benefit from using VRBE, we have to analyze IMPT plans using clinical results for additional patients and at other sites. Then, we also need to develop an RBE model to estimate accurately the VRBE. 


\section{Acknowledgements}

The research described in this paper was supported by grant number U19 CA021239 from the National Cancer Institute, and Cancer Prevention and Research Institute of Texas (RP160232). The University of Texas MD Anderson Cancer Center is supported in part by the National Institutes of Health/National Cancer Institute through Cancer Center Support Grant P30CA016672. We thank Sarah Bronson from the Department of Scientific Publications at MD Anderson for editing the manuscript.

\section{Conflicts of Interest}

The authors declare no conflicts of interest regarding the publication of this paper.

\section{References}

[1] Olsen, D.R., Bruland, O.S., Frykholm, G. and Norderhaug, I.N. (2007) Proton Therapy-A Systematic Review of Clinical Effectiveness. Radiotherapy Oncology, 83, 123-132. https://doi.org/10.1016/j.radonc.2007.03.001

[2] Mohan, R. and Grosshans, D. (2017) Proton Therapy-Present and Future. Advanced Drug Delivery Reviews, 109, 26-44.

https://doi.org/10.1016/j.addr.2016.11.006

[3] Lodge, M., Pijls-Johannesma, M., Stirk, L., Munro, A.J., De Ruysscher, D. and Jefferson, T. (2007) A Systematic Literature Review of the Clinical and Cost-Effectiveness of Hadron Therapy in Cancer. Radiotherapy Oncology, 83, 110-122. https://doi.org/10.1016/j.radonc.2007.04.007

[4] De Ruysscher, D., Lodge, M., Jones, B., Brada, M., Munro, A., Jefferson, T. and Pijls-Johannesma, M. (2012) Charged Particles in Radiotherapy: A 5-Year Update of a Systematic Review. Radiotherapy Oncology, 103, 5-7. https://doi.org/10.1016/j.radonc.2012.01.003

[5] Pedroni, E., Bacher, R., Blattmann, H., Copay, A., Lomax, A., Lin, S., Munkel, G., Scheib, S. and Schneider, U. (1995) The 200-MeV Proton Therapy Project at the Paul Scherrer Institute: Conceptual Design and Practical Realization. Medical Physics, 22, 37-53. https://doi.org/10.1118/1.597522

[6] Gillin, M.T., Sahoo, N., Bues, M., Ciangaru, G., Sawakuchi, G., Poenisch, F., Arjomandy, B., Martin, C., Titt, U., Suzuki, K., Smith, A.R. and Shu, X.R. (2010) Commissioning of the Discrete Spot Scanning Proton Beam Delivery System at the University of Texas M.D. Anderson Cancer Center, Proton Therapy Center, Houston. Medical Physics, 37, 154-163. https://doi.org/10.1118/1.3259742

[7] Kohno, R., Hotta, K., Dohmae, T., Matsuzaki, Y., Nishio, T., Akimoto, T., Tachikawa, T., Asaba, T., Inoue, J., Ochi, T., Yamada, M. and Miyanaga, H. (2017) Development of Continuous Line Scanning System Prototype for Proton Beam Therapy. International Journal of Particle Therapy, 3, 429-438. https://doi.org/10.14338/IJPT-16-00017.1

[8] Kooy, H.M. and Grassberger, C. (2015) Intensity Modulated Proton Therapy. British Journal of Radiology, 88, Article ID: 20150195. https://doi.org/10.1259/bjr.20150195

[9] Paganetti, H. (2014) Relative Biological Effectiveness (RBE) Values for Proton Beam Therapy. Variations as a Function of Biological Endpoint, Dose, and Linear Energy 
Transfer. Physics in Medicine and Biology, 59, R419-R472. https://doi.org/10.1088/0031-9155/59/22/R419

[10] Wedenberg, M. and Toma-Dasu, I. (2014) Disregarding RBE Variation in Treatment Plan Comparison May Lead to Bias in Favor of Proton Plans. Medical Physics, 41, Article ID: 091706-9. https://doi.org/10.1118/1.4892930

[11] Paganetti, H. (2015) Relating Proton Treatments to Photon Treatments via the Relative Biological Effectiveness-Should We Revise Current Clinical Practice? International Journal of Radiation Oncology Biology Physics, 91, 892-894. https://doi.org/10.1016/j.ijrobp.2014.11.021

[12] Giovannini, G., Bohlen, T., Cabal, G., Bauer, J., Tessonnier, T., Frey, K., Debus, J., Mairani A. and Parodi., K. (2016) Variable RBE in Proton Therapy: Comparison of Different Model Predictions and Their Influence on Clinical-Like Scenarios. Radiation Oncology, 11, 68. https://doi.org/10.1186/s13014-016-0642-6

[13] Oden, J., Eriksson, K. and Toma-Dasu, I. (2017) Inclusion of a Variable RBE into Proton and Photon Plan Comparison for Various Fractionation Schedules in Prostate Radiation Therapy. Medical Physics, 44, 810-822.

https://doi.org/10.1002/mp.12117

[14] Frese, M.C., Wilkens, J.J., Huber, P.E., Jensen, A.D., Oelfke, U. and Taheri-Kadkhoda, Z. (2011) Application of Constant vs. Variable Relative Biological Effectiveness in Treatment Planning of Intensity-Modulated Proton Therapy. International Journal of Radiation Oncology Biology Physics, 79, 80-88. https://doi.org/10.1016/j.ijrobp.2009.10.022

[15] Wilkens, J.J. and Oelfke, U. (2005) Optimization of Radiobiological Effects in Intensity Modulated Proton Therapy. Medical Physics, 32, 455-465. https://doi.org/10.1118/1.1851925

[16] Hong, L., Goitein, M., Bucciolini, M., Comiskey, R., Gottschalk, B., Rosenthal, S., Serago, C. and Urie, M. (1996) A Pencil Beam Algorithm for Proton Dose Calculations. Physics in Medicine and Biology, 41, 1305-1330. https://doi.org/10.1088/0031-9155/41/8/005

[17] Kohno, R., Takada, Y., Sakae, T., Terunuma, T., Matsumoto, K., Notomi, A. and Matsuda, H. (2003) Experimental Evaluation of Validity of Simplified Monte Carlo Method in Proton Dose Calculations. Physics in Medicine and Biology, 48, 1277-1288. https://doi.org/10.1088/0031-9155/48/10/303

[18] Hotta, K., Kohno, R., Takada, Y., Hara, Y., Tansho, R., Himukai, T., Kameoka, M.T., Nishio, T. and Ogino, T. (2010) Improved Dose-Calculation Accuracy in Proton Treatment Planning Using a Simplified Monte Carlo Method Verified with Three-Dimensional Measurements in an Anthropomorphic Phantom. Physics in Medicine and Biology, 55, 3545-3556. https://doi.org/10.1088/0031-9155/55/12/018

[19] Kohno, R., Cao, W., Yepse, P., Bai, X., Poenisch, F., Grosshans, D.R., Akimoto, T. and Mohan, R. (2017) Dose Comparison between Eclipse Dose Calculation and Fast Dose Calculator in Single- and Multi-Field Optimization Intensity-Modulated Proton Therapy Plans with Various Multi-Beams for Brain Cancer. International Journal of Medical Physics, Clinical Engineering and Radiation Oncology, 6, 421-432.

[20] Yepes, P., Randeniya, S., Taddei, P.J. and Newhauser, W.D. (2009) A Track Repeating Algorithm for Fast Monte Carlo Dose Calculations of Proton Radiotherapy. Nuclear Technology, 168, 334-337. https://doi.org/10.13182/NT09-A9298

[21] Yepes, P., Randeniya, S., Taddei, P.J. and Newhauser, W.D. (2009) Monte Carlo Fast Dose Calculator for Proton Radiotherapy: Application to a Voxelized Geometry Representing a Patient with Prostate Cancer. Physics in Medicine and Biology, 
54, N21-N28. https://doi.org/10.1088/0031-9155/54/1/N03

[22] Wilkens, J.J. and Oelfke, U. (2004) A Phenomenological Model for the Relative Biological Effectiveness in Therapeutic Proton Beams. Physics in Medicine and Biology, 49, 2811-2825. https://doi.org/10.1088/0031-9155/49/13/004

[23] Shaw, E., Kline, R., Gillin, M., Souhami, L., Hirschfeld, A., Dinapoli, R. and Martin, L. (1993) Radiation Therapy Oncology Group: Radio Surgery Quality Assurance Guidelines. International Journal of Radiation Oncology Biology Physics, 27, 1231-1239. https://doi.org/10.1016/0360-3016(93)90548-A

[24] Rechner, L.A., Howell, R.M., Zhang, R., Etzel, C., Lee, A.K. and Newhauser, W.D. (2012) Risk of Radiogenic Second Cancers Following Volumetric Modulated Arc Therapy and Proton Arc Therapy for Prostate Cancer. Physics in Medicine and Biology, 57, 7117-7132. https://doi.org/10.1088/0031-9155/57/21/7117

[25] Seco, J., Gu, G., Marcelos, T., Kooy, H. and Willers, H. (2013) Proton Arc Reduces Range Uncertainty Effects and Improves Conformality Compared with Photon Volumetric Modulated Arc Therapy in Stereotactic Body Radiation Therapy for Non-Small Cell Lung Cancer. International Journal of Radiation Oncology Biology Physics, 87, 188-194. https://doi.org/10.1016/j.ijrobp.2013.04.048

[26] Rah, J.E., Kim, G.Y., Oh, D.H., Kim, T.H., Kim, J.W., Kim, D.Y., Park, S.Y. and Shin, D. (2016) A Treatment Planning Study of Proton Arc Therapy for Para-Aortic Lymph Node Tumors: Dosimetric Evaluation of Conventional Proton Therapy, Proton Arc Therapy, and Intensity Modulated Radiotherapy. Radiation Oncology, 11, 140. https://doi.org/10.1186/s13014-016-0717-4

[27] Chen, Y. and Ahmad, S. (2012) Empirical Model Estimation of Relative Biological Effectiveness for Proton Beam Therapy. Radiation Protection Dosimetry, 149, 116-123. https://doi.org/10.1093/rpd/ncr218

[28] McNamara, A.L., Schuemann, J. and Paganetti, H. (2015) A Phenomenological Relative Biological Effectiveness (RBE) Model for Proton Therapy Based on All Published in Vitro Cell Survival Data. Physics in Medicine and Biology, 60, 8399-8416. https://doi.org/10.1088/0031-9155/60/21/8399

[29] Takada, K., Sato, T., Kumada, H., Koketsu, J., Taei, H., Sakurai, H. and Sakae, T. (2017) Validation of the Physical and RBE-Weighted Dose Estimator Based on PHITS Coupled with a Microdosimetric Kinetic Model for Proton Therapy. Journal of Radiation Research, 59, 1-9.

[30] Hojo, H., Dohmae, T., Hotta, K., Kohno, R., Motegi, A., Yagishita, A., Makinoshima, H., Tsuchihara, K. and Akimoto, T. (2017) Difference in the Relative Biological Effectiveness and DNA Damage Repair Processes in Response to Proton Beam Therapy According to the Positions of the Spread Out Bragg Peak. Radiation Oncology, 12, 111. https://doi.org/10.1186/s13014-017-0849-1

[31] Patel, D., Bronk, L., Guan, F., Peeler, R.C., Brons, S., Dokic, I., Abdollahi, A., Rittmüller, C., Jäkel, O., Grosshans, D., Mohan, R. and Titt, U. (2017) Optimization of Monte Carlo Transport Parameters and Validation of a Novel High Throughput Experimental Setup to Measure the Biological Effects of Particle Beams. Medical Physics, 44, 6061-6073. https://doi.org/10.1002/mp.12568

[32] Kohno, R., Sakae, T., Takada, Y., Matsumoto, K., Matsuda, H., Nohtomi, A., Terunuma, T. and Tsunashima, Y. (2002) Simplified Monte Carlo Dose Calculation for Therapeutic Proton Beams. Japan Journal of Applied Physics, 41, L294-L297. https://doi.org/10.1143/JJAP.41.L294

[33] Kohno, R., Takada, Y., Sakae, T., Terunuma, T., Matsumoto, K., Notomi, A. and Matsuda, H. (2003) Experimental Evaluation of Validity of Simplified Monte Carlo Method in Proton Dose Calculations. Physics in Medicine and Biology, 48, 
1277-1288. https://doi.org/10.1088/0031-9155/48/10/303

[34] Mizutani, S., Takada, Y., Kohno, R., Hotta, K., Tansho, R. and Akimoto, T. (2016) Application of Dose Kernel Calculation Using a Simplified Monte Carlo Method to Treatment Plan for Scanned Proton Beams. Journal of Applied Clinical Medical Physics, 17, 315-327. https://doi.org/10.1120/jacmp.v17i2.5747 